Journal of Innovative Image Processing (JIIP) (2019)

Vol.01/ No. 01

Pages: $39-50$

https://www.irojournals.com/iroiip/

DOI: https://doi.org/10.36548/jiip.2019.1.005

\title{
IMAGE INPAINTING TECHNIQUE FOR HIGH QUALITY AND RESOLUTION ENHANCED IMAGE CREATION
}

\author{
Dr. A. Pasumpon Pandian, \\ Professor, \\ Computer Science Engineering, \\ KGiSL Institute of Technology, \\ Coimbatore, India. \\ Email id: pasumponpandian32@gmail.com
}

\begin{abstract}
The image in-painting is the method of improving or enhancing the damaged and the missing parts of the images. This process would be very essential preprocessing procedure in case of the medical image analysis for the diagnosis of the disease. The traditional ways of in-painting being ineffective the paper proposes hybrid image in-painting technique combining the edge connect, patch match and the deep image prior for the images to improve the quality and the resolution of the images, the proposed method is tested with different number of images from the gathered form the website to prove the competence of the proposed image inpainting technique.
\end{abstract}

Keywords: Image In- painting, Image Restoration, Image Enhancement, object removal, filling the gap

\section{INTRODUCTION}

The image in painting or the restoration is basically a process of making up the areas that are damaged or missing in an image. [12] The main scope of the image restoration is usually a method of reconstructing the original image from its noisy state. The image restoration or the in painting may be the final result for certain application and the preprocessing stage in many other applications. For e.g. when the image transmitted through a channel is affected by the noise, or when the image is superimposed with the sophisticated and complex patterns, or texts or any other objects [9]. It is also viewed as the art of adjusting the images such that they cannot be recognized by the ordinary observer [10] the author Guillemot, in his paper address the image in painting as the very complex problem or an "ill posed inverse problem that has no appropriate solutions, and points out the necessity of the prior information of the images to enhance the resolution of the images. Among many methods available Guillemot [12] in his paper projects certain image in-painting techniques they are (i) diffusion based in painting, (ii) variational in painting, and (iii) exemplar based in painting. Janarthanan [11] presenting the survey of the available techniques for the image in painting, says that image in-painting are very much useful in the red eye correction as well as compression etc. The image in-painting on the other hand can be viewed as the "removal of image defects such as the scratches and the blotches" the fig .1 below shows the images where the in-painting techniques can be applied. 
Journal of Innovative Image Processing (JIIP) (2019)

Vol.01/ No. 01

Pages: $39-50$

https://www.irojournals.com/iroiip/

DOI: https://doi.org/10.36548/jiip.2019.1.005
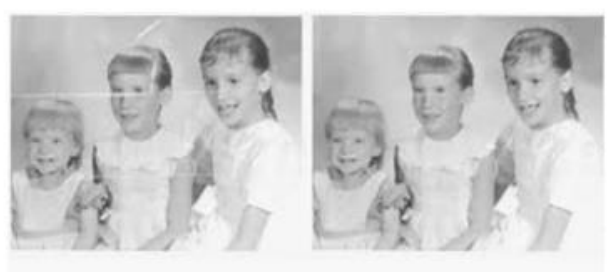

(a)
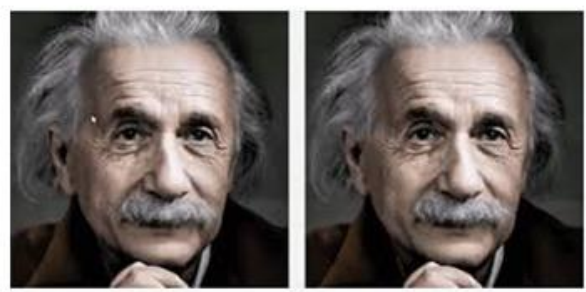

(c)

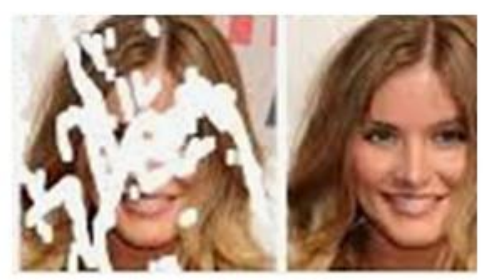

(b)

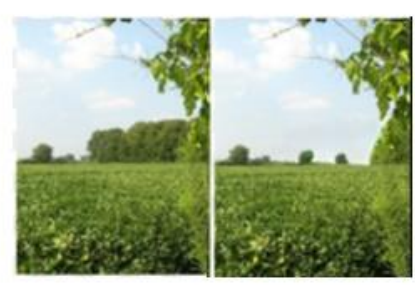

(d)

Fig .1 images where in-painting are applied, (a) improve the resolution of the images, (b) restore the damaged image, (c) improves the quality of the image (d) object removal

The paper exploits the hybridized image in-painting technique incorporating the edge connect, patch match and the Deep image prior for the image in-painting (image restoration and enhancement) to have a high quality and enhanced resolution images.

The remaining of the paper is organized is with the related works in the section II, proposed work in the section III, results in the section IV, conclusion in the section $\mathrm{V}$

\section{RELATED WORKS}

Bertalmio, et al [1], and Luminita et al [3] the author elaborates the need of image in-painting in the images and puts forward an automatic image restoration algorithm, for filling in the gaps in the images that belong to various back ground concurrently. 
Journal of Innovative Image Processing (JIIP) (2019)

Vol.01/ No. 01

Pages: $39-50$

https://www.irojournals.com/iroiip/

DOI: https://doi.org/10.36548/jiip.2019.1.005

Richard et al [2] "the author presents the simple in-painting algorithm for the rebuilding of the minute missing and the damaged portions of the images." The author states that the proffered method in the paper is "two or three orders of magnitude faster in comparison to the prevailing methods.

Yamauchi et al [4] the author combines the "texture synthesis and the image in -painting, to restore the images and enhance the resolution of the images".

Criminisi, et al [5] the author proposes the texture and structure based effective algorithm for the removing a large objects and filling in the gaps. The effectiveness of the algorithm is tested using the synthetic and the real images.

Wong et al [6] the "paper proposes a new strategy of exemplar image in-painting utilizing the non-local image information from multiple samples and picking the samples used based on the underlying image content, to achieve an enhanced visual quality. This method is combined with the existing exemplar in-painting to have an enhanced performance."

Dobrosotskaya et al [7] the author incorporates the "basic geometric frames of the diffuse interface methods and the non-locality of wavelets to remove the blurry edges, preserve the usage of the fast solvers, and the creation of the technique to extend from the binary images to the grayscale". "The wavelet based image in painting is done in the paper for the grayscale images in painting"

Daribo et al [8] the paper utilizes the "depth image based rendering technique" along with the "preprocessing techniques and the in painting approach based on the structural and the texture propagation to address the disocclusion problem in the $3 \mathrm{D}$ video service, and to fill in the gaps.

Xie, et al [9] the paper organizes the "task of image denoising and the blind in painting combining the sparse coding and the deep networks pre-trained with the denoising auto-encoder." The method proffered enables the removal of the text or another image that are superimposed on the images. "The training scheme utilized in the paper is efficient and enhances the unsupervised feature learning"

Bugeau et al [10] the author incorporates the three building blocks such as the "copy-paste texture synthesis (CPTS), geometric PDE and the coherence among the neighboring pixels, to afford an algorithm for the in painting for the images with the minimum energy functional." 
Journal of Innovative Image Processing (JIIP) (2019)

Vol.01/ No. 01

Pages: $39-50$

https://www.irojournals.com/iroiip/

DOI: https://doi.org/10.36548/jiip.2019.1.005

Janarthanan, et al [11] and Pandya, et al [13]the paper is a survey about the image in-painting techniques such as the (i) texture synthesis based in-painting, (ii) PDE based in-painting, exemplar based in-painting, (iii) semi-automatic and (iv) fast in-painting techniques along with the highlights on the limitations and the short comings encountered in each technique.

Guillemot, et al [12] the author shows the different algorithms that are available for the image in-painting. He describes the image restoration methods along with the challenges in it and presents the subjective assessment to analyze whether the restored images are visually pleasing and physically plausible.

Qin et al [14] the author proposes a "novel joint data hiding and the compression scheme for the digital images utilizing the side matching vector quantization and the image in-painting to embedded with the secret data and compress the images.

Modha, et al [15] "the paper deals with the automatic in-painting based image restoration next to the text identification and the removal of the text form the images" this is done by detecting the exact location of the text in the image , removing them and replacing the removed gaps with the in-painting methods.

Chhabra, et al [16] the author presents the detailed survey on the exemplar based image in-painting techniques for the enhancing the quality of the image, the author describes the techniques along with the limitations found in the each technique.

Nazeri et al [17] the author presents the "Generative image in painting with adversarial edge learning" to improve the quality of the by providing a proper connection to the edges.

Barnes et al [18] the author exploits the patch match to edit the image and improve the quality and the resolution of the image.

Ulyanov et al [19] the author uses the deep convolutional neural networks for enhancing the image quality and the resolution by having the prior knowledge of the images form the huge set of the examples images. 
Journal of Innovative Image Processing (JIIP) (2019)

Vol.01/ No. 01

Pages: $39-50$

https://www.irojournals.com/iroiip/

DOI: https://doi.org/10.36548/jiip.2019.1.005

\section{PROPOSED WORK}

The image restoration or the in-painting is an essential preprocessing stage especially in the medical imaging, the method of in painting has become very important among the medical image that are been acquired from the MRI (Magnetic Resonance Imaging ), CT ( Computed Tomography) Scan etc. The image in-painting is also applied to the images in the photographs that are aged, or damaged due to the flood, fire or any other occurrence. The traditional techniques for the image in-painting being very time consuming, costly and with the result images of low resolution and reduced quality, the paper puts forward a hybridized technique of image in-painting to improve the quality as well as the resolution of the images.

The proposed method combines the edge connect [17] patch match [18] and the deep image prior [19] to improve the quality of the image and enhance its resolution. The fig. 2 below shows the flow in the proposed process to achieve a high quality image with enhanced resolution.

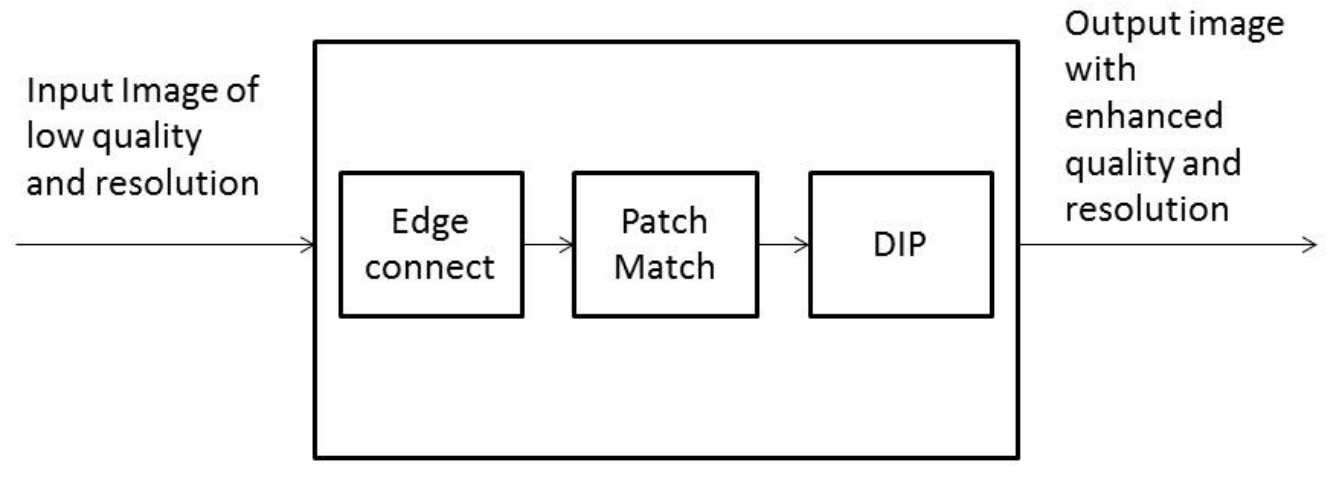

Fig .2 Proposed Flow Process

ISSN: 2582- 4252 
Journal of Innovative Image Processing (JIIP) (2019)

Vol.01/ No. 01

Pages: $39-50$

https://www.irojournals.com/iroiip/

DOI: https://doi.org/10.36548/jiip.2019.1.005

As shown in the fig. 2 the images to be restored are subjected to the hybridized technique that incorporates the edge connect, patch match and the deep prior image. The image that is to be in-painted is subjected to the each technique one after the other to have an enhanced image quality and resolution. The initial stage that the image is subjected is the Edge Connect and that is followed by the patch match and then finally deep image prior is applied to have better final results.

3.1. EDGE CONNECT [17]: The method is utilized to find the appropriate edges of the missed regions in the images utilizing the edge generator. The edge generator provides the edges for the missing regions in the images. The edge generator receives the grayscale images as the input and generates the mask for the image with 1 for the areas that are missing and the image with 0 for the back ground of the images. The generator then predicts the edge map of $t$ the region that is masked. The network being trained with the feature mapping and the adversarial losses compares for the losses in the in-between layers of the discriminator and predicts the edges of the missing portion. The fig below shows the output obtained from the edge generator.

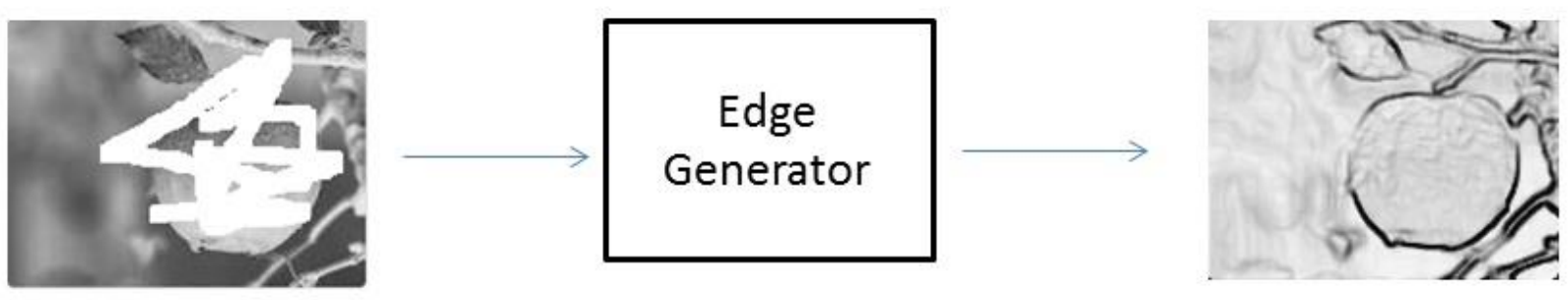

Fig .3 Output of Edge Generator

3.2. PATCH MATCH [18]: The patch match enables the speedy identification of the matches of the image patches; this finds the appropriate nearest neighbor matches among the image patches via random sampling and the natural coherence. The high quality patches for the missing region of the image could be identified with the maximum number of iterations. The patch match proceeds in three stages in the initial stage the random offsets are filled in the nearestneighbor field followed by the next stage the proliferation of the optimal patches an iterative process for finding the adjacent pixel, the final stage is the random search for deciding with the optimized best solutions. The fig.4below shows the results of the patch matching for the input image fed.

ISSN: $2582-4252$ 


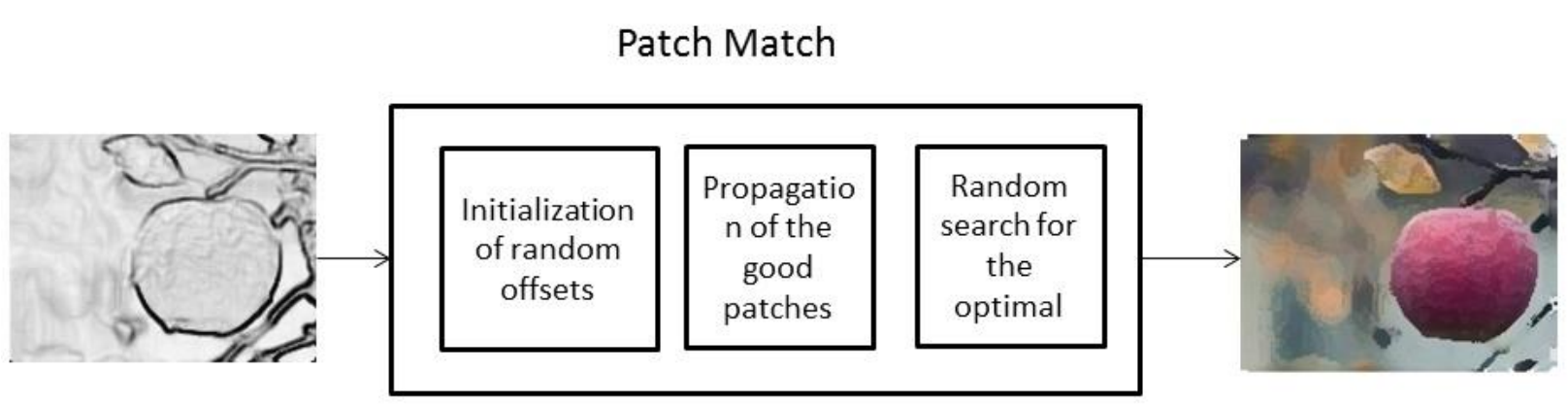

Fig .4 Output of the Patch Match

3.3. DEEP IMAGE PRIOR [19]: To further improve the image qualities the edge-connect and the patch match is followed by the deep image prior to further enhance the quality and the resolution of the image. The deep image prior that is done with the deep convolutional neural network, for improving the quality of the image and to make it more presentable for the next stage, the method of deep image prior up samples the image that has to be restored with the convolutional network utilizing the structure of the image as the image prior. The technique ensures a super resolution with cleaner and sharper edges images even without learning. The image generated by the decoder networks is subjected to the deep network and follows a pre-restoration, refinement and the restoration to get an in-painted image. The method iteratively exploits the image prior and reduces the mean square error in the masked regions and achieves a high quality and a resolution enhanced image at the output. The fig .5 shows the high resolution output achieved at the

Deep Image Prior

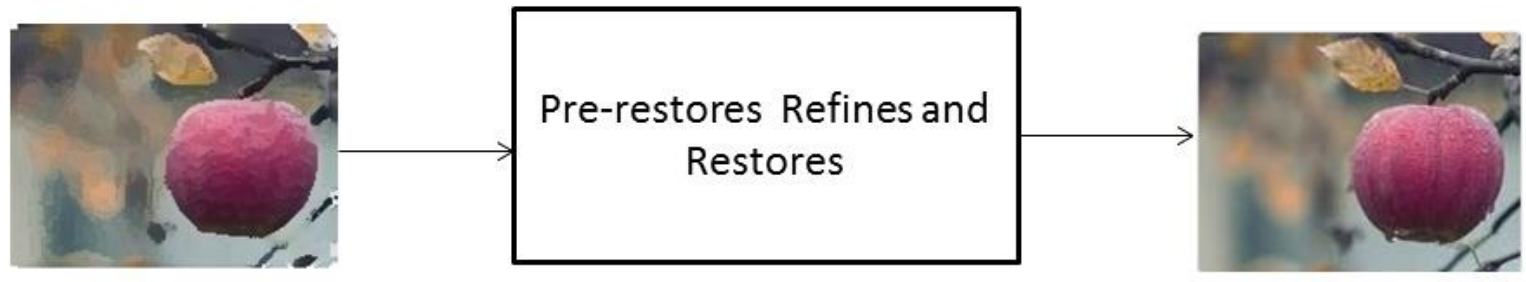

Fig .5 Output of the Deep Image Prior 
Journal of Innovative Image Processing (JIIP) (2019)

Vol.01/ No. 01

Pages: $39-50$

https://www.irojournals.com/iroiip/

DOI: https://doi.org/10.36548/jiip.2019.1.005

The hybridized image in-painting technique incorporating the three methods the edge-connect, patch match and the deep image prior ensures a creation of an image with improved quality and resolution.

\section{RESULTS AND DISCUSSION}

The proposed technique of hybridized image in-painting technique was test over many images gathered from the website to ensure the competence of the proposed method. The images that are to be restored are fed as input or uploaded as shown below.

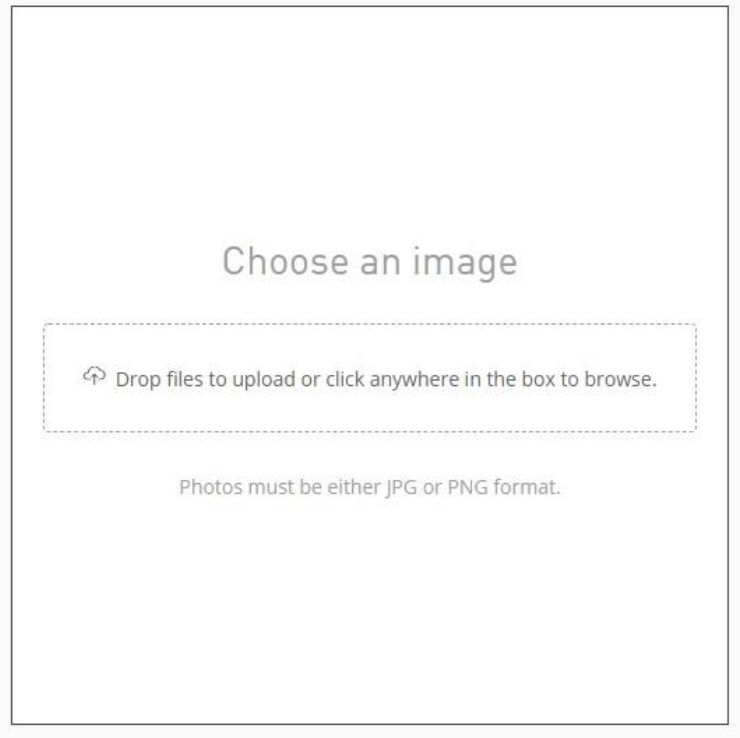

Fig .6. The Input Image Window

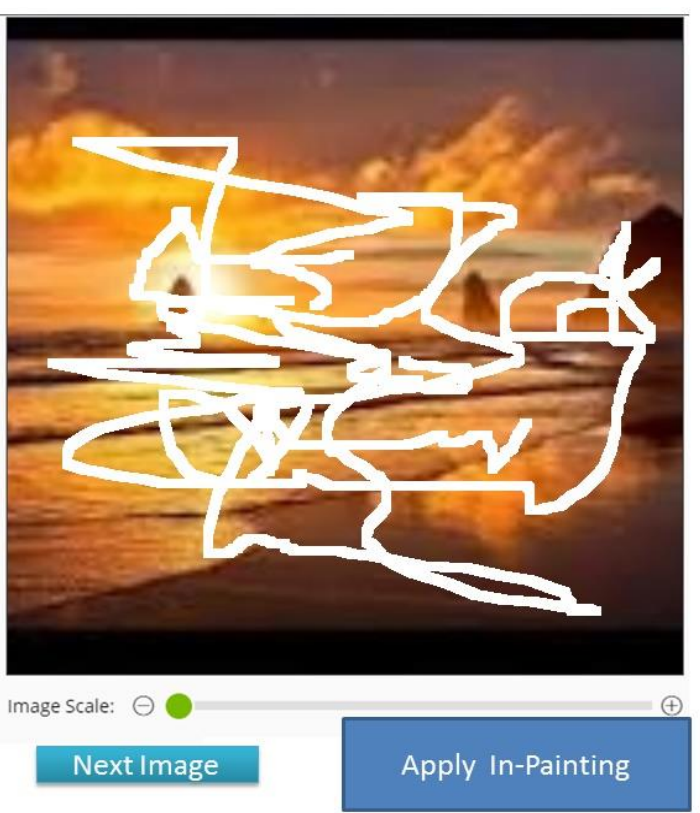

Fig.7 Input Image Fed 
Journal of Innovative Image Processing (JIIP) (2019)

Vol.01/ No. 01

Pages: $39-50$

https://www.irojournals.com/iroiip/

DOI: https://doi.org/10.36548/jiip.2019.1.005

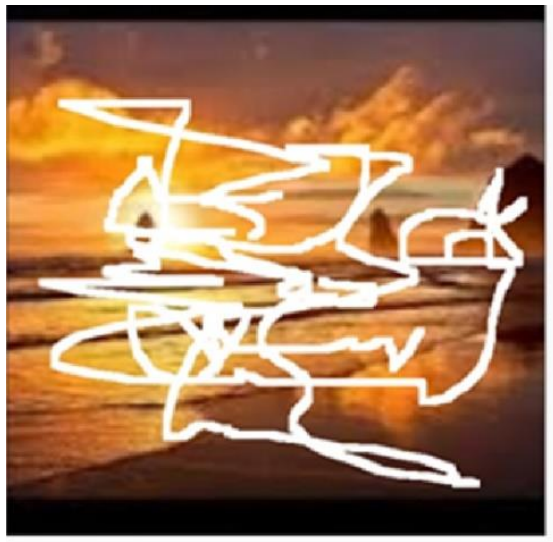

ORIGINAL IMAGE

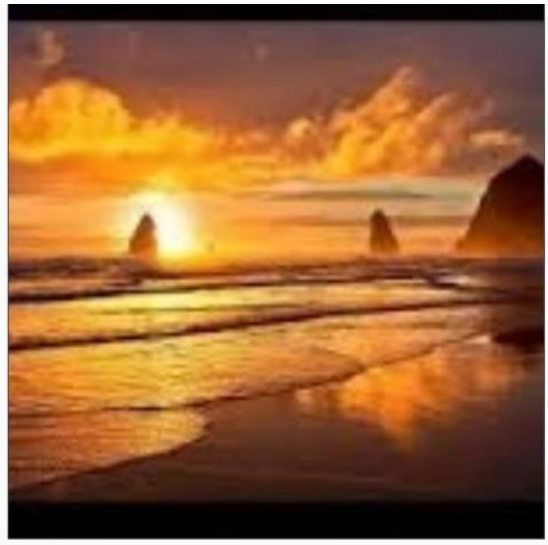

INPAINTED RESULT

Fig .8 The In-painted Images

The above steps shows the image in-painting using the hybridized image in-painting technique that incorporates the edge- connect, patch match and the deep prior image. The result obtained shows the competence of the proposed method. The quality of the proposed image in-painting technique combing the three techniques is validated by measuring the SSIM, and the PSNR of the proposed along with the state of art approaches such as the partial convolution and the patch offset, edge connect, and patch match. The end result attained with the hybridized techniques is more effective compared to the other methods.

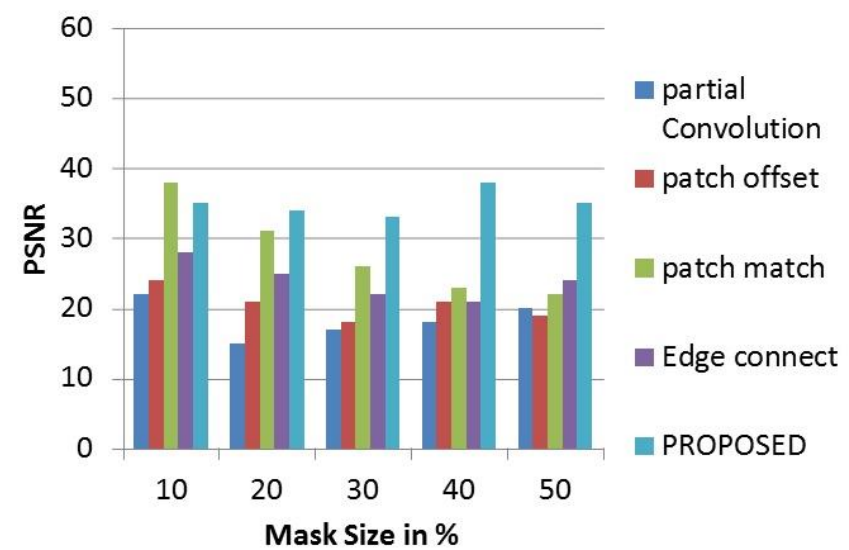

Fig .9 PSNR 
Journal of Innovative Image Processing (JIIP) (2019)

Vol.01/ No. 01

Pages: $39-50$

https://www.irojournals.com/iroiip/

DOI: https://doi.org/10.36548/jiip.2019.1.005

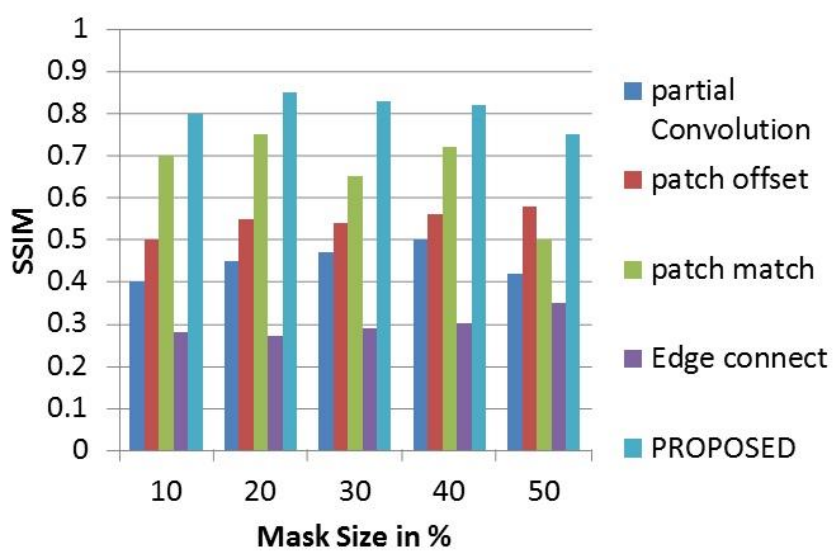

Fig.10 SSIM

The measurement of the performance metrics show that the proposed hybridized technique provide a much enhanced image quality and resolution than the state art approaches such as the partial convolution and the patch offset, edge connect, and patch match.

\section{CONCLUSION}

The paper proposes the hybridized in-painting model that in-corporates the edge-connect, patch match and the deep image prior to restore the damaged images and enhance the quality and the resolution of the images proceeds with the following steps initiated with the edge generator, that produces the edge of the missing areas, followed by the patch match that does the random search of the optimal patches among the images for the missing areas, and finally produced to the DIP that enhances the quality of the image with the cleaned and sharpened edges improving the resolution of the image. The proposed hybridized frame work is tested with the damaged image obtained from the website; the quality of the proposed method is validated by measuring the PSNR and the SSIM along with the other sate art of approaches such as the partial convolution etc. the results shows the competence of the proffered method in creating image with improved quality and resolution. In future the paper is to continue with the implementation of the proposed method for enhancing the quality of the MRI Scans for the medical imaging. 
Journal of Innovative Image Processing (JIIP) (2019)

Vol.01/ No. 01

Pages: $39-50$

https://www.irojournals.com/iroiip/

DOI: https://doi.org/10.36548/jiip.2019.1.005

\section{REFERENCES}

[1] Bertalmio, Marcelo, Guillermo Sapiro, Vincent Caselles, and Coloma Ballester. "Image inpainting." In Proceedings of the 27th annual conference on Computer graphics and interactive techniques, pp. 417424. ACM Press/Addison-Wesley Publishing Co., 2000.

[2] Richard, Manuel M. Oliveira Brian Bowen, and McKenna Yu-Sung Chang. "Fast digital image inpainting." In Appeared in the Proceedings of the International Conference on Visualization, Imaging and Image Processing (VIIP 2001), Marbella, Spain, pp. 106-107. 2001.

[3] Bertalmio, Marcelo, Luminita Vese, Guillermo Sapiro, and Stanley Osher. "Simultaneous structure and texture image inpainting." IEEE transactions on image processing 12, no. 8 (2003): 882-889.

[4] Yamauchi, Hitoshi, Jörg Haber, and H-P. Seidel. "Image restoration using multiresolution texture synthesis and image inpainting." In Proceedings Computer Graphics International 2003, pp. 120-125. IEEE, 2003.

[5] Criminisi, Antonio, Patrick Pérez, and Kentaro Toyama. "Region filling and object removal by exemplarbased image inpainting." IEEE Transactions on image processing 13, no. 9 (2004): 1200-1212.

[6] Wong, Alexander, and Jeff Orchard. "A nonlocal-means approach to exemplar-based inpainting." In 2008 15th IEEE International Conference on Image Processing, pp. 2600-2603. IEEE, 2008.

[7] Dobrosotskaya, Julia A., and Andrea L. Bertozzi. "A wavelet-Laplace variational technique for image deconvolution and inpainting." IEEE Transactions on Image Processing 17, no. 5 (2008): 657-663.

[8] Daribo, Ismael, and Béatrice Pesquet-Popescu. "Depth-aided image inpainting for novel view synthesis." In 2010 IEEE International Workshop on Multimedia Signal Processing, pp. 167-170. IEEE, 2010.

[9] Xie, Junyuan, Linli Xu, and Enhong Chen. "Image denoising and inpainting with deep neural networks." In Advances in neural information processing systems, pp. 341-349. 2012.

[10] Bugeau, Aurélie, Marcelo Bertalmío, Vicent Caselles, and Guillermo Sapiro. "A comprehensive framework for image inpainting." IEEE Transactions on Image Processing 19, no. 10 (2010): 2634-2645.

[11] Janarthanan, V., and G. Jananii. "A detailed survey on various image inpainting techniques." Bonfring International Journal of Advances in Image Processing 2, no. 2 (2012): 01-03.

[12] Guillemot, Christine, and Olivier Le Meur. "Image inpainting: Overview and recent advances." IEEE signal processing magazine 31, no. 1 (2013): 127-144.

[13] Pandya, Nirali, and Bhailal Limbasiya. "A survey on image inpainting techniques." International Journal of Current Engineering and Technology 3, no. 5 (2013): 1828-1831.

[14] Qin, Chuan, Chin-Chen Chang, and Yi-Ping Chiu. "A novel joint data-hiding and compression scheme based on SMVQ and image inpainting." IEEE transactions on image processing 23, no. 3 (2013): 969-978.

[15] Modha, Uday, and Preeti Dave. "image inpainting-Automatic Detection and Removal of Text from images." International Journal of Engineering Research and Applications (IJERA), ISSN (2014): 2248-9622. 
Journal of Innovative Image Processing (JIIP) (2019)

Vol.01/ No. 01

Pages: $39-50$

https://www.irojournals.com/iroiip/

DOI: https://doi.org/10.36548/jiip.2019.1.005

[16]Chhabra, Jaspreet Kaur, and Mr Vijay Birchha. "Detailed survey on exemplar based image inpainting techniques." International Journal of Computer Science and Information Technologies 5, no. 5 (2014): 6350635.

[17] Nazeri, Kamyar, Eric Ng, Tony Joseph, Faisal Qureshi, and Mehran Ebrahimi. "Edgeconnect: Generative image inpainting with adversarial edge learning." arXiv preprint arXiv:1901.00212 (2019).

[18]Barnes, Connelly, Eli Shechtman, Adam Finkelstein, and Dan B. Goldman. "PatchMatch: A randomized correspondence algorithm for structural image editing." In ACM Transactions on Graphics (ToG), vol. 28, no. 3, p. 24. ACM, 2009.

[19] Ulyanov, Dmitry, Andrea Vedaldi, and Victor Lempitsky. "Deep image prior." In Proceedings of the IEEE Conference on Computer Vision and Pattern Recognition, pp. 9446-9454. 2018. 